\title{
Facets of Subjective Health Horizons Are Differentially Linked to Brain Volume
}

\author{
Sandra Düzel ${ }^{1}$, Johanna Drewelies², Denis Gerstorf², Ilja Demuth ${ }^{3}$, Simone Kühn ${ }^{1,4}$, \\ and Ulman Lindenberger ${ }^{1,5,6}$ \\ ${ }^{1}$ Center for Lifespan Psychology, Max Planck Institute for Human Development, Berlin, Germany \\ 2 Department of Psychology, Humboldt University Berlin, Berlin, Germany \\ ${ }^{3}$ Charité - Universitätsmedizin Berlin, corporate member of Freie Universität Berlin, Humboldt-Universität zu Berlin, \\ and Berlin Institute of Health, Germany, Department of Endocrinology and Metabolism \\ ${ }^{4}$ Department of Psychiatry and Psychotherapy, University Clinic Hamburg-Eppendorf, Hamburg, Germany \\ ${ }^{5}$ Max Planck UCL Centre for Computational Psychiatry and Ageing Research, Berlin, Germany \\ ${ }^{6}$ European University Institute, San Domenico di Fiesole (FI), Italy
}

\begin{abstract}
An active lifestyle including physical exercise and novelty processing is considered to promote brain health. Also, subjective future time perspectives (FTP) are known to shape motivation and goal-directed behavior, with links to objective health, well-being, and cognition. Nevertheless, the links between subjective FTP and brain physiology are largely unknown. We report data from 326 healthy older adults who completed the Subjective Health Horizon Questionnaire (SHH-Q) and structural magnetic resonance imaging (MRI). Voxel-based morphometry analyses revealed associations between (1) the SHH-Q Novelty factor and brain regions of the episodic memory network, and (2) the SHH-Q Body factor and regions contributing to the cortical representation of bodily states. Longitudinal and experimental data are needed to better understand the etiology of these links.
\end{abstract}

Keywords: Berlin Aging Study II, BASE-II, future time perspective, FTP, active successful aging, Subjective Health Horizon Questionnaire (SHHQ), brain structure

Engagement in an active and stimulating lifestyle is crucial to successful cognitive and brain aging (Düzel, Bunzeck, Guitart-Masip, \& Düzel, 2010; Lindenberger, 2014; Lindenberger, Wenger, \& Lövdén, 2017). One key factor toward that end is that people are motivated to select physically or cognitively challenging life goals and to translate these goals into corresponding lifestyles (Hertzog, Kramer, Wilson, \& Lindenberger, 2008).

Prominent lifespan psychological theories have long been advanced and empirically tested to better understand how motivational focus, behavior, and future time horizons work together (Brandtstädter \& Rothermund, 2002; Heckhausen, Wrosch, \& Schulz, 2010). For example, it is well established that individual differences in future time perspectives (FTP) are correlated with key outcomes in the domains of physical health, cognitive functioning, and well-being (Kozik, Hoppmann, \& Gerstorf, 2014; Mather \& Carstensen, 2005; Mather \& Schoeke, 2011). At the same time, we are only beginning to understand whether and how specific dimensions of people's FTP are associated with their brain functioning and integrity.

\section{The Nature and Correlates of Dimensions of Subjective Health} Horizon

The FTP is one component of personal lifetime concerned with how individuals look at their future, as opposed to their present or past (Brandtstädter \& Rothermund, 2002; Brandtstädter, Rothermund, Kranz, \& Kühn, 2010). FTP can be defined as the perceptions and expectations people have with regard to their future, specifically the extent to which they perceive the future as open-ended and offering opportunities - or as being limited and closed in nature. In this context, prior work used the Future Time Perspective Scale (Carstensen \& Lang, 1996) to capture individuals' global perceptions of their overall time left to live. It was found that a more extended FTP is positively related to emotion (Lang \& Carstensen, 2002), health (Kozik et al., 2014), and social cognition (Mather \& Carstensen, 2003, 2005). In our view, a questionnaire that assesses FTP for an active and stimulating lifestyle should take into account that FTP may not form a unidimensional construct and may show different temporal extensions or limitations, e.g., an older person with a limited physical future time horizon may still be motivated for future cognitive stimulating activities that do not require bodily fitness. 
Following from notions about the multidimensionality of people's FTP (e.g., Brandtstädter \& Greve, 1994), we recently developed the Subjective Health Horizon Questionnaire (SHHQ; Düzel et al., 2016) in order to fully account for people's ability to simulate and imagine their own future, by assessing four interrelated but distinct dimensions of FTP of an active lifestyle. In order to index how long people anticipate pursuing different facets of an active lifestyle in the future, we validated a latent four-factor model of the SHH-Q by applying a confirmatory latent factor analysis resulting in four distinct but correlated facets of activity-related FTP: SHH-Q Body, tapping into expectations regarding one's future bodily fitness, SHH-Q Novelty, referring to novelty-oriented exploration in one's future as well as SHH-Q Work, encompassing aspects of occupational goals in the future, and SHH-Q Life Goals, comprising the future perspective of overall goals in life. The latter two served as reference variables. Further, we found that two of these factors, namely, SHH-Q Body and SHH-Q Novelty, showed differential associations with objective measures of physical health and episodic memory (Düzel et al., 2016), respectively. More specifically, people who reported a wide time horizon on the SHH-Q Body dimension had a better metabolic health status. In contrast, a long FTP on the SHH-Q Novelty dimension was associated with particularly good performance in tasks tapping into the episodic memory domain.

\section{This Study}

Previous findings of individual differences in the SHH-Q Novelty and SHH-Q Body facets suggested that these FTP dimensions were uniquely associated with episodic memory performance and metabolic health status (Düzel et al., 2016). These findings are noteworthy because they not only highlight the multidimensionality of FTP, but also emphasize the importance of FTP of two main lifestyle activities - cognitive and physical stimulation - that have been previously shown to modulate cognitive trajectories across adulthood (Rowe \& Kahn, 2015).

To illustrate, numerous studies showed that improvements in physical fitness and cognitive stimulation can ameliorate brain structure and brain function as well as enhance cognitive performance in adulthood (Lindenberger, 2014; Van Praag, Kempermann, \& Gage, 2001). As a consequence, one could expect that FTP dimensions would also relate to specific brain regions.

In the current study, we take previous research on the FTP dimensionality one step further by investigating whether certain FTP dimensions, namely, imagining oneself exploring novel environments or learning new contents (as assessed by the SHH-Q Novelty factor) and imagining oneself as being physically active (as assessed by the SHH-Q Body factor) may also relate to variations in gray matter structures in specific brain regions.

We hypothesize that the FTP for engaging in novel and cog- nitively stimulating activities (SHH-Q Novelty) depends on the the brain structures previously linked to episodic memory: (1) because individuals may use current episodic memory abilities as a reference point to estimate their future abilities to learn novel information (e.g., how well they orient in unknown environments or remember the names of new people); (2) because the episodic memory system may support the ability to imagine oneself in future behaviors related to exploring novel environments (e.g., learning a new language or meeting new people). Previous research suggests that components of the core episodic memory system, namely, the prefrontal cortex, the parietal cortex (e.g., posterior cingulate cortex), the medial temporal lobe (e.g., hippocampus), and the retrosplenial cortex are critical to episodic memory, that is, the ability to memorize and recall novel events, but also for the ability to imagine future scenarios (e.g., Schacter, Addis, \& Buckner, 2008).

In contrast, the FTP for physical health may rely on different brain regions. Specifically, we hypothesize that engaging with novel information does not necessary require physical activity (e.g., learning a new language or reading a new book), and, in turn, physical activity is not always related to exposure to novel information and can be carried out in a familiar environment (e.g., exercise at home on a bicycle ergometer). Hence, its FTP may be more strongly rooted in current bodily health and not depend on episodic memory performance.

The current study aims to explore these differences on the neural basis in greater detail. To test our hypothesis, we make use of data obtained from a subsample of healthy older participants in the Berlin Aging Study II (BASE-II; Bertram et al., 2014) who underwent magnetic resonance imaging (MRI). We applied voxel-based morphometry analyses (VBM; Ashburner \& Friston, 2000) in the MRI subsample to examine whether morphological variations in gray matter brain regions are specifically associated with SHH-Q Body and SHH-Q Novelty factors. VBM is becoming increasingly widely used as a tool to examine patterns of brain structure and change in healthy aging (e.g., Good et al., 2001) and neuroanatomical correlates of behavior (e.g., Maguire et al., 2003).

\section{Methods}

\section{Participants and Study Design}

Older participants in the larger BASE-II study ranged in age from 61 to 88 years $(n=1,371$; mean $=70.1 ; S D=3.78 ; 50.9 \%$ female), and were drawn from BASE-II. Participants provided informed consent in accord with the Declaration of Helsinki. Individuals participated in two cognitive testing sessions scheduled 1 week apart and were tested in small groups (about 6 participants per group). Each session lasted about $3.5 \mathrm{~h}$. A digitalized version of the SHH-Q was administered during the first 
cognitive session. From one session to the next, participants were asked to fill out psychosocial questionnaires related to subjective health and well-being. Eligible participants were invited to a structural brain imaging session within a mean time interval of 3.2 months after completing the cognitive testing.

From the initial MRI sample of 340 participants with MRI, we excluded 14 participants because of missing data on MRI, the SHH-Q, or the covariates. The final sample retained for the current analysis included 326 older adults (mean age $=69.7$, $S D=3.9$ years). Sample demographics showed a greater proportion of men $(61.7 \%)$ than women $(38.3 \%)$, and a mean level of years of education approaching 1 year of university (mean = $14.1, S D=2.9$ years). BASE-II participants in the MRI cohort did not differ from those who did not undergo MRI scanning in terms of age, sex, subjective health, and cognition. The Ethics Committee of the Max Planck Institute for Human Development approved the cognitive assessment, and the Ethics Committee of the German Society for Psychology (DGPs) additionally approved the MRI protocol. Participants provided written informed consent and received monetary compensation for their participation in BASE-II and the MRI study.

\section{Measures}

The Subjective Health Horizon Questionnaire (SHH-Q, Düzel et al., 2016) was administered during the first cognitive session of BASE-II (single items of the SHH-Q are listed in the Appendix 2) in a digital version, independently of other psychosocial constructs. In order to avoid confounds, other psychosocial constructs had been obtained in a take-home questionnaire that was handed out at the end of the first session. For the current study, we used two factors of the SHH-Q: The Body dimension (SHH-Q Body, e.g., "Up to which age do you believe you will be able to maintain or even increase your fitness by training?"), and the Novelty dimension (SHH-Q Novelty, e.g., "Up to which age do you feel physically and mentally able to come to grips with new technology independently?") were both assessed with 9 items. Reliabilities for the subscales were good (Cronbach's $\alpha \geq .89$ ). In order to assign higher SHH-Q scores to older individuals reporting a difference between estimated age and chronological age, responses to each item were adjusted by calculating the difference between individuals' actual age and their estimated future ages, and scaling by the ratio of actual age to maximum lifetime (defined as 110 years). The adjustment reflects the assumption that a time perspective of, say, 15 years expressed at age 75 (the resulting age-adjusted score would be 10.2 years) extends expected vitality into an older age than a time perspective of 15 years expressed at age 65 (the resulting age-adjusted score would be 8.8 years). All follow-up analyses are based on these age-adjusted scores, and lower scores on both scales indicate a more limited time horizon in either future lifestyle dimension.

\section{Covariates}

\section{The Future Time Perspective (FTP) Scale}

We used the 10-item Future Time Perspective Scale (Carstensen \& Lang, 1996), assessed as part of the take-home psychosocial assessment questionnaire between the two cognitive sessions. Participants responded to each item (e.g., "Many opportunities await me in the future.") using a Likert scale ranging from 1 (very untrue for me) to 7 (very true for $m e$ ). The scale composite consists of the unit-weighted mean across items, with lower scores indicating a more limited general future time horizon. Reliability was acceptable (Cronbach's $\alpha=.88$ ).

\section{Sociodemographic Variables}

Participants' age was calculated for the date on which the first cognitive session took place and the psychosocial questionnaire was handed out. Sex was indexed as a dichotomous variable.

\section{Total Intracranial Volume (TIV)}

A well-known source of individual differences in total and regional brain volume is the between-person variation in head size (Mathalon, Sullivan, Rawles, \& Pfefferbaum, 1993), often measured by TIV. For example, some of the differences between the sexes in brain volume can be accounted for by differences in TIV (e.g., Whitwell, Crum, Watt, \& Fox, 2001). We used the probabilistic tissue class images derived from CAT12 segmentation to give tissue volume estimates, with TIV simply being the sum of gray matter, white matter, and CSF voxels.

\section{Objective Health}

Physical fitness represents one's state of general muscle strength, musculoskeletal capacity, and general vitality, and has been indexed repeatedly by grip strength and forced expiratory volume (Infurna \& Gerstorf, 2013). In this study, we used continuous information on grip strength and forced expiratory volume as indicators of physical fitness. Grip strength was measured with a dynamometer (Smedley, ranging from 0 to $100 \mathrm{~kg}$ ). Participants started with the dominant followed by the nondominant hand, and were asked "to grasp with as much force as possible." Three measurements for each hand were taken, with the highest value of each hand being selected for later analysis. Forced expiratory volume in one second (FEV1) was used as an overall indicator of lung function. We only analyzed spirometric measurements with sufficient measurement quality, fully in line with standard procedures.

\section{Magnetic Resonance Imaging (MRI) Acquisition}

\section{Scanning Procedure}

The structural images were acquired on a Siemens TIM Trio 3T scanner (Erlangen, Germany) using a 32-channel head coil 
to increase the signalto-noise ratio to improve image quality. The T1 images were obtained using a three-dimensional T1weighted magnetization prepared gradient-echo sequence (MPRAGE) based on the ADNI protocol (www.adni-info.org) (repetition time $(\mathrm{TR})=2500 \mathrm{~ms}$; echo time $(\mathrm{TE})=4.77 \mathrm{~ms}$; $\mathrm{TI}$ $=1100 \mathrm{~ms}$, acquisition matrix $=256 \times 256 \times 176$, flip angle $=$ $7^{\circ} ; 1 \times 1 \times 1 \mathrm{~mm}$ voxel size) .

\section{VBM Preprocessing and Analyses}

Structural data was processed by means of the CAT12 toolbox (http://dbm.neuro.uni-jena.de/cat12/CAT12-Manual.pdf) implemented in SPM12 (http://www.fil.ion.ucl.ac.uk/spm/software/spm12/) using the suggested preprocessing default parameters. Preprocessing steps incorporated intrasubject realignment, bias correction, segmentation, and spatial normalization. After an initial realignment of individual anatomical scans to standardized (MNI) space, the mean of the realigned images was calculated and used as reference image in a subsequent realignment. The realigned individual images were then bias-corrected to account for signal inhomogeneities. The mean image was segmented into gray matter, white matter, and cerebrospinal fluid and normalized using DARTEL. The resulting spatial normalization parameters were then applied to the segmentations of the bias-corrected individual images of both time points, which were again realigned. To allow comparison of the absolute tissue volume, voxel values were modulated using the Jacobian determinants (i.e., linear and nonlinear components) derived from the spatial normalization. Finally, the modulated gray matter images were smoothed with a Gaussian kernel of $8 \mathrm{~mm}$ FWHM.

Statistical analysis was carried out by means of total brain correlation of gray matter volume and two regression models containing the SHH-Q Body and SHH-Q Novelty factor scores as dependent variables separately. Age, sex, the FTP score, and the subject's TIV were entered as covariates. The resulting maps were thresholded with $p<.001$ and the statistical extent threshold was used to correct for multiple comparisons combined with a nonstationary smoothness correction based on permutation as proposed by Hayasaka and Nichols (2004). This is necessary because VBM data exhibit spatially varying smoothness; we used nonstationary cluster size tests in our analysis as well as a relatively high cluster-forming threshold, $p$ $=.001$, on images smoothed with a $6 \mathrm{~mm}$ Gaussian kernel. Nonstationary tests were carried out using Hayasaka's nonstationary toolbox for SPM (http://fmri.wfubmc.edu/cms/NS-General). The nonstationary toolbox corrects for expected variation in cluster size in nonstationary images under the null hypothesis, using statistical random field theory.

\section{Data Analysis}

\section{Factor Scores of the SHH-Q}

In order to minimize Type 1 errors, we carefully selected the variables and covariates according to our hypothesis and ap- plied rigorous data reduction by using factor analyses before conducting the VBM analyses. The individual two SHH-Q factor scores were estimated using the regression method (modal posterior estimator) in Mplus v6.1 (Muthén \& Muthén, 2010) on the basis of the four-latent factor model reported in Düzel and colleagues (2016). Next, we applied the extracted SHH-Q factor scores as independent variables in two separate wholebrain VBM multiple regression analyses of the MR sample only.

\section{VBM - Second Level Modeling and Contrasts}

We aimed to examine neuroanatomical correlates of the two SHH-Q dimensions using multiple regression analysis. The first model included the SHH-Q Body factor score, while age, sex, FTP, and TIV volume of each participant were included as covariates and thus regressed out. The second model included the SHH-Q Novelty factor score with identical covariates.

\section{Results}

\section{Demographic Information}

Table 1 reports intercorrelations for the variables under study. Of note is that older adults reported more constrained health horizons on both SHH-Q Body $(r=-.37)$ and SHH-Q Novelty $(r=-.36)$ dimensions, whereas the age correlation was substantially smaller for the FTP scale $(r=-.17)$. In addition, both SHH$\mathrm{Q}$ dimensions only moderately intercorrelated with the FTP scale $(r=.46$, all $p$ 's $<.001)$, suggesting that the two sets of questionnaires tap into partly different aspects of the larger measurement space. Both objective health indicators showed no association with SHH-Q Body and SHH-Q Novelty factors (all $p$ 's $>.05)$. Grip strength was significantly associated with $\operatorname{sex}(r=.78)$ and total brain volume $(r=.49$, see Table 1$)$.

\section{Voxel-Based Morphometry (VBM)}

The first VBM analysis investigating the association between SHH-Q Body and gray matter regions showed positive associations with voxels in the right superior frontal gyrus (BA 10; peak voxel: $\mathrm{x}=19.5, \mathrm{y}=64.5, \mathrm{z}=16.5 ; p<.001)$, right middle frontal gyrus (BA $8 \& 9$; peak voxel: $\mathrm{x}=30, \mathrm{y}=45, \mathrm{z}=13.5$; $p<.001$ ), left fusiform gyrus (BA 20) and parahippocampal gyrus (BA 36; peak voxel: $\mathrm{x}=-45, \mathrm{y}=-28.5, \mathrm{z}=-30.0 ; p<$ .001 ), the left posterior Insula (BA 13; peak voxel: $x=-37.5, y$ $=-21, \mathrm{z}=22.5 ; p<.001)$, the left superior temporal gyrus (BA 41; peak voxel: $\mathrm{x}=-45, \mathrm{y}=-34.5, \mathrm{z}=10.5 ; p<.001$ ), the left superior motor area (BA 6; peak voxel: $x=-16, y=-7.5$, $\mathrm{z}=73 ; p<.001$ ) and the right inferior parietal lobe (BA 39; peak voxel: $\mathrm{x}=33, \mathrm{y}=-57, \mathrm{z}=46.5 ; p<.001)$. All significant 
Table 1. Descriptive statistics and intercorrelations for all variables under study

\begin{tabular}{|c|c|c|c|c|c|c|c|c|c|c|}
\hline & \multicolumn{10}{|c|}{ Intercorrelations } \\
\hline & Mean & $S D$ & 01. & 02. & 03. & 04. & 05. & 06. & 07. & 08. \\
\hline 01. Age & 70.1 & 3.8 & 1 & & & & & & & \\
\hline 02. Sex (1 = men) & 1.51 & .49 & -.02 & 1 & & & & & & \\
\hline 03. FTP (1-6) & 2.62 & .68 & $-.17 *$ & -.12 & 1 & & & & & \\
\hline 04. Total brain volume & 1941.7 & 165.2 & .03 & $-.65^{\star \star}$ & -.06 & 1 & & & & \\
\hline 05. Grip strength & 32.25 & 8.5 & .00 & $-.78 * \star$ & .02 & $.49 * *$ & 1 & & & \\
\hline 06. FEV1 & .75 & .08 & -.06 & -.03 & .03 & -.03 & .02 & 1 & & \\
\hline 07. SHH-Q Novelty FS & 0.200 & 2.15 & $-.36 * \star$ & -.16 & $.46 * \star$ & .07 & .06 & -.01 & 1 & \\
\hline 08. SHH-QBody FS & 0.245 & 1.97 & $-.37 \star \star$ & -.01 & $.46 * *$ & .07 & .01 & .00 & $.87 * \star$ & 1 \\
\hline
\end{tabular}

Note. FTP = Future time perspective. FEV1 = Forced expiratory volume in one second. SHH-QNovelty FS = Factor score for the Novelty dimension of the Subjective Health Horizon Questionnaire. SHH-QBody FS = Factor score for the Body dimension of the Subjective Health Horizon Questionnaire. Significant values are printed in bold. $N=326 .{ }^{*} p<.05,{ }^{*} p<.001$.

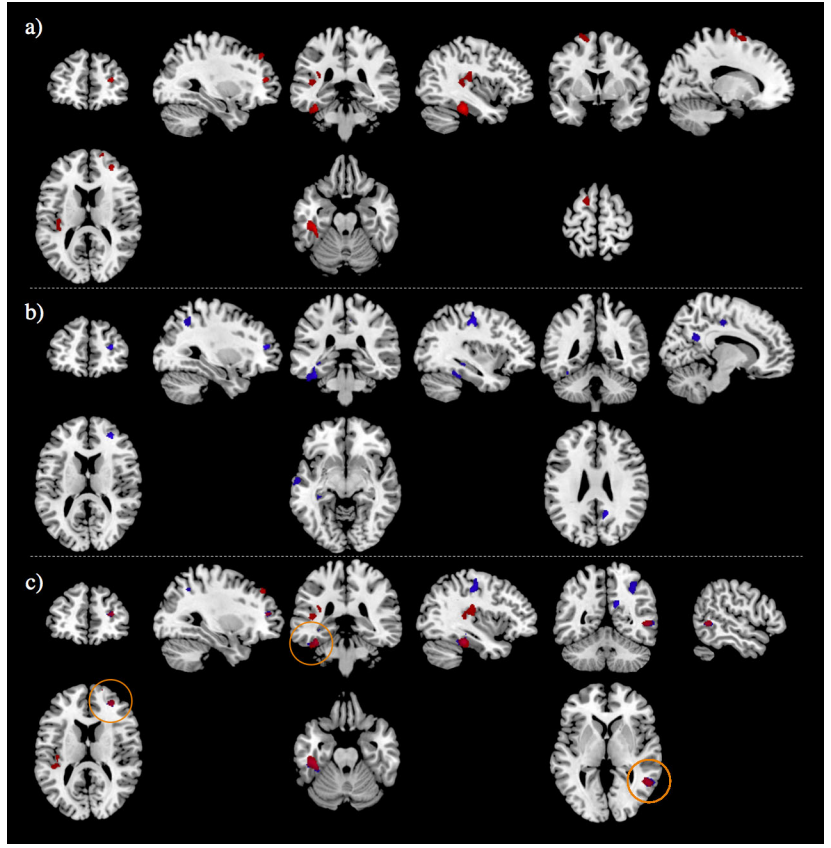

Figure 1. Distinct brain regions resulting from two VBM analyses containing the (a) SHH-Q Body factor score as variable of interest: TSL; I; SMA and (b) the SHH-Q Novelty factor score: HC; MFG, RSC, PG, SMA, whereas (c) illustrates overlapping regions detected in both analyses; SFG, FG, PHG, MTG, SPL ( $p=.001$, corrected for multiple comparisons).

Note. (1) TSL: temporal superior lobe (BA 41); I: insula (BA 13); SMA: superior motor area (BA 6); (2) HC: hippocampus; MFG: middle frontal gyrus (BA 6); RSC: retrosplenial cortex (BA 31); PG: precentral gyrus (BA 4); SMA: superior motor area (BA 24); (3) SFG: superior frontal gyrus (BA 10); FG: fusiform gyrus (BA 20); PHG: parahippocampal gyrus (BA 36); MTG: middle temporal gyrus (BA 21); SPL: superior parietal lobe (BA 40); all p's = .001, corrected for multiple comparisons.

clusters are corrected for multiple comparisons, cluster extent threshold $k>100$.

Interestingly, the second VBM, which included the SHH-Q Novelty, revealed a significant positive association in core areas of the EM network, namely, the right superior frontal gyrus
(BA 10; peak voxel: $\mathrm{x}=30, \mathrm{y}=45, \mathrm{z}=13 ; p<.001)$, left fusiform gyrus (BA 20) and parahippocampal gyrus (BA 36; peak voxel: $\mathrm{x}=-40.5, \mathrm{y}=-30, \mathrm{z}=-21 ; p<.001)$, in the right retrosplenial cortex (peak voxel: $\mathrm{x}=13.5, \mathrm{y}=-52.5, \mathrm{z}=27 ; p<.001$ ), the bilateral regions of the pre- and postcentral lobe (right: BA $3 \& 4$; peak voxel: $\mathrm{x}=-34.5, \mathrm{y}=-18, \mathrm{z}=46.5 ; p<.001 /$ left: $\mathrm{BA3} \& 4$; peak voxel: $\mathrm{x}=33, \mathrm{y}=-57, \mathrm{z}=46.5 ; p<.001)$ and the right inferior parietal lobe (BA 39; peak voxel: $\mathrm{x}=33, \mathrm{y}=-57, \mathrm{z}=$ 46.5; $p<.001)$ after controlling for FTP, age, sex, and total brain volume (Figure 1b). Figure 1c illustrates overlapping regions detected in both analyses: superior frontal gyrus (BA 10); fusiform gyrus (BA 20); parahippocampal gyrus (BA 36); middle temporal gyrus (BA 21) and superior parietal lobe (BA 40).

\section{Discussion}

We investigated how interindividual differences in imagination of physical and cognitive stimulating future lifestyle choices are related to structural brain volume among older adults. Our findings suggest that different dimensions of an active lifestyle in one's future, as indexed by the two subscales of the SHH-Q, relate to both common and unique brain regions.

In particular, our main findings indicate that the SHH-Q Novelty dimension is associated with core brain regions of the episodic memory network, whereas the SHH-Q Body dimension is associated with the insula, a brain region that, among other functions, establishes cortical representations of physiological conditions. Such differential brain associations are striking when considering that the two SHH-Q Body and SHH-Q Novelty subscales are highly intercorrelated (see Table 1).

The first set of VBM findings revealed that a wider time perspective for future-oriented engagement in novel activities (SHH-Q Novelty) is associated with core components of the 
episodic memory network, including the right superior frontal gyrus, the left fusiform and parahippocampal gyrus, the posterior hippocampus, as well as the right retrospenial cortex. Additionally, bilateral regions of the precentral lobe clusters emerged in this model.

Among other functions, findings of several functional neuroimaging studies showed that imagining the future as well as remembering the past (subjective mental time travel) requires widely distributed neural regions including the aforementioned regions such as the prefrontal cortex (Szpunar, Watson, \& McDermott, 2007; Wheeler, Stuss, \& Tulving, 1997), hippocampus (Hassabis, Kumaran, \& Maguire, 2007; Hassabis, Kumaran, Vann, \& Maguire, 2007), parietal cortex (Arzy, Collette, Ionta, Fornari, \& Blanke, 2009), and retrosplenial regions (Buckner, Andrews-Hanna, \& Schacter, 2008). Interestingly, these core regions are also implicated in the novelty-processing hierarchy (Lisman, Grace, \& Duzel, 2011).

The medial temporal lobe (including hippocampus, fusiform, and parahippocampal regions) has been suggested to reflect the critical role in retrieving episodic memories of the past (Benoit \& Schacter, 2015) and thus might serve as a source to evaluate past behavior related to experiences of novel lifestyle choices. Moreover, the hippocampus is crucial for the processing and consolidation of new memories (e.g., Düzel et al., 2010; Lisman \& Grace, 2005), and facilitates predictions of the future (Schacter et al., 2012).

Additionally, imagining novelty exposure and/or exploration in the future has been shown to activate both, the hippocampus as well as the midbrain, which may be one plausible mechanism of inducing a motivational state promoting future exploratory behavior (Bulganin \& Wittmann, 2015; Wittmann, Bunzeck, Dolan, \& Düzel, 2007). The role of the midbrain in novelty-related FTP needs to be further investigated.

The retrospenial cortex is a core hub within the EM network and showed a specific association with SHH-Q Novelty factor. This is an interesting finding because its reciprocal connection to the dorsolateral prefrontal cortex, parietal and occipital cortex, and hippocampus in animals suggests its role in episodic memory (Vann, Aggleton, \& Maguire, 2009). Patients with damage involving the retrospenial cortex consistently show problems in acquiring new information (Maguire et al., 2003) and selective deficits in spatial orientation.

From a clinical point of view, the association between the hippocampus, the fusiform, and parahippocampal gyrus and retrospenial cortex with the SHH-Q Novelty factor is also interesting with regard to the progression of age-related memory dysfunction into minimal cognitive impairment (MCI), a prodromal stage of Alzheimer's disease (Nestor, Fryer, Smielewski, \& Hodges, 2003). This is because both regions are among the first to show structural and functional disturbance in MCI and may also affect the extension of the SHH-Q Novelty dimension.

The second VBM analyses revealed that a more extended FTP on the SHH-Q Body dimension was associated with larger gray matter density in the left posterior insula, a region that, among other functions, establishes the cortical representation of the current physiological conditions of the entire body ("interoception"). Perceptions of the body provide a sense of one's current physical condition and also underlie mood and emotional states. The interoceptive representation of distinct and highly resolved feelings from the body include pain, temperature, itch, sensual touch, muscular and visceral sensations, vasomotor activity, hunger, and thirst, and are engendered in the anterior insular cortex, which may provide one possible reference for the subjective image of the current state of the material self (Craig, 2002, 2003; Di Lernia, Serino, \& Riva, 2017). These findings suggest that individual differences in the gray matter density of the insula may serve as a source for the subjective estimation of one's current bodily health status - and may thus serve as a source (among others) for the subjective estimation of FTP of being physically active. Additional significant clusters emerged within the right superior frontal gyrus, right middle frontal gyrus, and regions of the left medio-temporal lobe - brain regions that, in contrast, are known to be involved in processing subjective time and are discussed in the next paragraph (Nyberg, Kim, Habib, Levine, \& Tulving, 2010).

In both VBM analyses, gray matter density in brain regions in prefrontal areas and the medio-temporal cortex showed overlapping associations with the SHH-Q Body as well as the SHH$\mathrm{Q}$ Novelty factor. Interestingly, these regions have consistently been reported in functional brain-imaging (fMRI) studies as being related to future thinking, goal planning, and the processing of subjective time (Hassabis, Kumaran, \& Maguire, 2007; Hassabis \& Maguire, 2007; Nyberg et al., 2010). In the context of FTP, the right prefrontal cortex appears to be particularly important in the discrimination of long time intervals, consistent with the results of studies showing that damage to the prefrontal cortex impairs the discrimination of longer durations more than that of short durations (Droit-Volet, 2013). Additionally, several patient studies suggest that individuals with damage to the prefrontal cortex have problems thinking about their continued existence (Wheeler et al., 1997). More recently, findings suggest that this region is engaged during self-referential processing (Hassabis, Kumaran, Vann et al., 2007) as well as during imaginary scene construction (Addis, Wong, \& Schacter, 2007). These cognitive processes may also be important for establishing an overall FTP of one's own future health and cognitive performance. However, the precise function of this region in relation to future time is not yet understood.

Taken together, the first VBM analyses revealed that the perceived ability to explore novel settings in the future (SHH-Q Novelty) is associated with core components of the episodic memory system, that is known to process novelty and support the exploration of novel environments. In contrast, we found different brain regions being associated with the future perspectives regarding the ability to remain physically active (SHH-Q Body). The results demonstrate that individual differences in these two domains of FTP are variously associated with the structural integrity of specific brain regions. These findings are 
reference

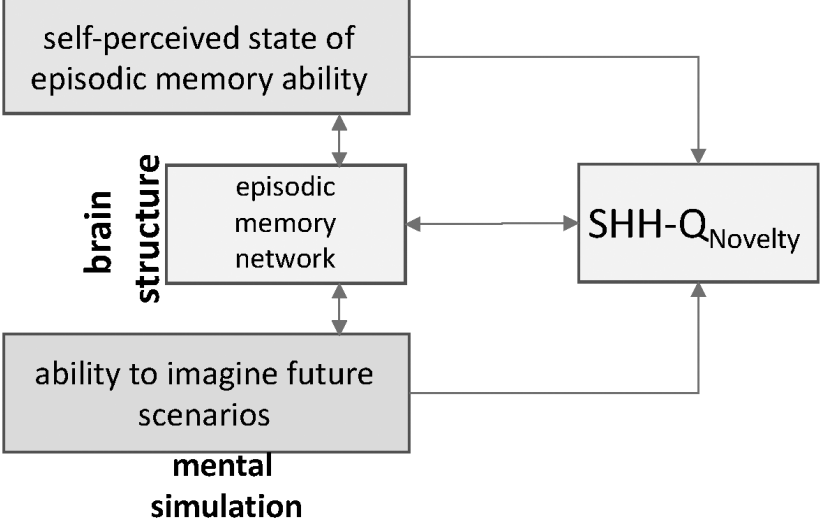

Figure 2. Tentative framework linking FTP of cognitive stimulating activities (the $\mathrm{SHH}-\mathrm{Q}$ Novelty) to brain structure.

striking as they are in line with our previous behavioral findings showing that individuals distinguish between physical and cognitive aspects (Düzel et al., 2016) when judging different lifestyle aspects of their FTP.

Figure 2 summarizes this assumption in a first tentative framework for the SHH-Q Novelty dimension, suggesting that the FTP for engaging in novel and cognitively stimulating activities may depend on the integrity of brain regions of the episodic memory system for two reasons: First, individuals may use their current episodic memory performance as a subjective reference in order to estimate of how long they will be able, e.g., to learn novel information, how well they will orient in unknown environments, or remember the names of new people. Second, the integrity of these brain networks may support the ability to imagine oneself in future behaviors related to exploring novel environments, learning a new language, or meeting new people, which has been shown in several studies (Hassabis, Kumaran, \& Maguire, 2007; Hassabis \& Maguire, 2007; Nyberg et al., 2010).

\section{Limitations and Outlook}

The limitations of the present study are first and foremost that its results are based on cross-sectional observations that do not provide direct information about individual differences in change. In particular, longitudinal observations and experimental interventions are needed to probe the psychological mechanisms underlying the construction of FTP as well as the embedding of these mechanisms into biological and social systems of influence. Moreover, several components of this network show age-related decline in normal aging (Raz \& Lindenberger, 2013; Raz \& Rodrigue, 2006), and we would like to investigate whether age-related decline in these regions might narrow the subjective experience of future time horizons for stimulating cognitive and novel activities. It will be intriguing to examine whether the neural underpinnings found here are specific to people engaging in thinking about their health in the future or whether they can also be found when people consider their present or past health and cognitive state. These longitudinal investigations are planned within BASE-II in the very near future.

In addition, the current study aimed to investigate how the SHH-Q factors are linked to the neural level resulting in selection of the same covariates as used in our initial study for the VBM analyses. Nevertheless, including other covariates such as measures of intelligence or socioeconomic status may be important to explore the strength of associations found in more detail. Regarding the strong links between subjective time horizons and age, which we took into account by age-adjusted scaling of each SHH-Q item, it would be interesting to explore which component of the $\mathrm{SHH}$ is more strongly related to selfperception of aging in future research.

Second, the validity of the SHH-Q Work and Life Goals subscales remains to be examined more closely in future analyses. The lack of significant associations of the Work and Life Goals subscales of the SHH-Q in a follow-up VBM analysis (see Appendix 1) requires further investigation, and the putative associations of these two subscales with socioeconomic and social factors, which were not fully explored in the present study, need to be examined in greater detail. Here, specific neuromodulatory networks such as the dopaminergic or emotion-regulatory systems may play a role; however, they cannot be fully assessed by using exploratory VBM analyses. Other MR techniques which were not part of the present study are required, but need to be applied in follow-up studies.

In follow-up analyses we intend to examine the role played by connectivity between specific brain regions as well as white matter integrity (obtained by resting state and diffusion tensor imaging). One assumption from our findings is that participants reporting a more extended FTP for novel, cognitively stimulating activities also show a higher connectivity between brain regions of the episodic memory network.

Additionally, it needs to be kept in mind that BASE-II involves a convenience sample. As a partial remedy to this limitation, BASE-II has been linked to data from the German Socio-Economic Panel (SOEP), which uses a far more representative sampling scheme than BASE-II. Systematic comparisons of BASE-II data with SOEP data allow for estimates of selection bias and offer statistical tools for bias correction. As research with the SHH-Q unfolds in the context of BASE-II and in other studies, issues of selective sampling, replicability, and generalizability need to be addressed. Moreover, a person's preference for certain future perspectives regarding an enriched and active lifestyle may be influenced by other factors and differ across cultures. A next step would be to investigate whether the four-factor model of the SHH$\mathrm{Q}$ can be replicated across different cultures and how the associations found in the previous and current study change across cohorts and cultures. In collaboration with the University of Barcelona we intend to assess the SHH-Q in a Spanish sample and thus address some of the open questions in the near future.

Finally, we used VBM as one neuroimaging technique. When interpreting the results regarding its biological meaning it must 
be taken into consideration that it is an indirect method of measuring gray matter density. Further, different MRI techniques may uncover specific underlying mechanisms of the associations between brain regions and different factors of the SHH-Q. To gain more insight into the role of the brain regions, the next step would be to design an fMRI experiment in which participants are ask to imagine their past, present, or future selves or to respond to SHH-Q items while in the scanner. This approach would give a clear insight into the function of brain regions while shifting through different dimensions of future lifestyle activities.

\section{Conclusions}

Taken together, our results provide novel evidence about the underlying neural correlates of time perspective by taking into account the multidimensional facets and differences in individual future time profiles. The results of the present study indicate that specific brain regions known to process subjective time (Nyberg et al., 2010) are associated with two main future time facets, namely, activities related to novelty exploration, which assesses future engagement in cognitive stimulation, and bodily fitness, which relates to future physical health and fitness activities. Interestingly, the VBM results revealed differences in neural correlates of the two future time dimensions, including distinct areas for the SHH-Q Novelty and SHH-Q Body future time factor, suggesting that the scales are sensitive enough to be used in this context.

These findings support our overarching assumption that people's future time horizons are shaped by a multitude of different factors, including its well-studied psychosocial characteristics, but that they also - as reported here - rely on neurobiological factors. Moreover, these findings may help in designing interventions to enhance the motivation to engage in brain-related lifestyle choices.

\section{Author Notes}

This manuscript reports data from the Berlin Aging Study II (BASE-II). The BASE-II research project (Co-PIs are Lars Bertram, Denis Gerstorf, Ulman Lindenberger, Graham Pawelec, Elisabeth Steinhagen-Thiessen, and Gert G. Wagner) was supported by the German Federal Ministry of Education and Research (Bundesministerium für Bildung und Forschung, BMBF) under grant numbers \#16SV5536K, \#16SV5537, \#16SV5538, and \#16SV5837, and \#01UW0808). Another source of funding is the Max Planck Institute for Human Development, Berlin, Germany. Further details about the study can be obtained at https://www.base2.mpg.de/en

\section{Editorial Note}

Acting Editor was Frieder R. Lang.

\section{Declaration of Conflicts of Interest}

The authors declare that no conflicts of interest exist.

\section{References}

Addis, D., Wong, A., \& Schacter, D. (2007). Remembering the past and imagining the future: Common and distinct neural substrates during event construction and elaboration. Neuropsychologia, 45, 1363-1377. doi 10.1016/j.neuropsychologia. 2006.10.016

Arzy, S., Collette, S., Ionta, S., Fornari, E., \& Blanke, O. (2009). Subjective mental time: The functional architecture of projecting the self to past and future. The European Journal of Neuroscience, 30, 2009-2017. doi 10.1111/j.1460-9568.2009.06974.x

Ashburner, J., \& Friston, K. J. (2000). Voxel-based morphometry: The methods. Neurolmage, 11, 805-821. doi 10.1006/nimg.2000.0582

Benoit, R., \& Schacter, D. (2015). Specifying the core network supporting episodic simulation and episodic memory by activation likelihood estimation. Neuropsychologia, 75, 450-457. doi 10.1016/j.neuropsychologia.2015.06.034

Bertram, L., Böckenhoff, A., Demuth, I., Düzel, S., Eckardt, R., Li, S.C. C., ... Steinhagen-Thiessen, E. (2014). Cohort profile: The Berlin Aging Study II (BASE-II). International Journal of Epidemiology, 43, 703-712. doi 10.1093/ije/dyt018

Brandtstädter, J., \& Greve, W. (1994). The aging self: Stabilizing and protective processes. Developmental Review, 52-80. doi 10.1006/drev.1994.1003

Brandtstädter, J., \& Rothermund, K. (2002). The life-course dynamics of goal pursuit and goal adjustment: A two-process framework. Developmental Review, 22, 117-150. doi 10.1006/drev.2001.0539

Brandtstädter, J., Rothermund, K., Kranz, D., \& Kühn, W. (2010). Final decentrations - personal goals, rationality perspectives, and the awareness of life's finitude. European Psychologist, 15, 152-163. doi 10.1027/1016-9040/a000019

Buckner, R., Andrews-Hanna, J., \& Schacter, D. (2008). The brain's default network: Anatomy, function, and relevance to disease. Annals of the New York Academy of Sciences, 1124, 1-38. doi 10.1196/annals.1440.011

Bulganin, L., \& Wittmann, B. (2015). Reward and novelty enhance imagination of future events in a motivational-episodic network. PLOS ONE, 10(11), e0143477. doi 10.1371/journal.pone.0143477

Carstensen, L., Lang, \& Lang, FR. (1996). Future Time Perspective Scale. Stanford, CA: Stanford University Press.

Craig, A. D. (2002). How do you feel? Interoception: The sense of the physiological condition of the body. Nature Reviews Neuroscience, 3, 655-666. doi 10.1038/nrn894

Craig, A. D. (2003). Interoception: The sense of the physiological condition of the body. Current Opinion in Neurobiology, 13, 500-505.

Di Lernia, D., Serino, S., \& Riva, G. (2017). Pain in the body: Altered interoception in chronic pain conditions. A systematic review. Neuroscience and Biobehavioral Reviews, 71, 328-341. doi 10.1016/j.neubiorev.2016.09.015

Droit-Volet, S. (2013). Time perception, emotions and mood disorders. Journal of Physiology, 107, 255-264. doi 10.1016/ j.jphysparis.2013.03.005 
Düzel, E., Bunzeck, N., Guitart-Masip, M., \& Düzel, S. (2010). NOvelty-related Motivation of Anticipation and exploration by Dopamine (NOMAD): Implications for healthy aging. Neuroscience and Biobehavioral Reviews, 34, 660-669. doi 10.1016/j.neubiorev. 2009.08.006

Düzel, S., Voelkle, M. C., Düzel, E., Gerstorf, D., Drewelies, J., Steinhagen-Thiessen, E., ... Lindenberger, U. (2016). The Subjective Health Horizon Questionnaire (SHH-Q): Assessing future time perspectives for facets of an active lifestyle. Gerontology, 62, 345-353. doi 10.1159/000441493

Good, C. D., Johnsrude, I. S., Ashburner, J., Henson, R. N., Friston, K.J., \& Frackowiak, R.S. (2001). A voxel-based morphometric study of aging in 465 normal adult human brains. Neurolmage, 14, $21-36$. doi 10.1006/nimg. 2001.0786

Hassabis, D., Kumaran, D., \& Maguire, E. (2007). Using imagination to understand the neural basis of episodic memory. The Journa of Neuroscience, 27, 14365-14374. doi 10.1523/jneurosci.454907.2007

Hassabis, D., Kumaran, D., Vann, S., \& Maguire, E. (2007). Patients with hippocampal amnesia cannot imagine new experiences. Proceedings of the National Academy of Sciences of the United States of America, 104, 1726-1731. doi 10.1073/pnas.0610561104

Hassabis, D., \& Maguire, E. (2007). Deconstructing episodic memory with construction. Trends in Cognitive Sciences, 11, 299-306. doi 10.1016/j.tics.2007.05.001

Hayasaka, S., \& Nichols, T. E. (2004). Combining voxel intensity and cluster extent with permutation test framework. Neurolmage, 23, 54-63. doi 10.1016/j.neuroimage.2004.04.035

Heckhausen, J., Wrosch, C., \& Schulz, R. (2010). A motivational theory of lifespan development. Psychological Review, 117, 32-60. doi 10.1037/a0017668

Hertzog, C., Kramer, A., Wilson, R., \& Lindenberger, U. (2008). Enrichment effects on adult cognitive development: Can the functional capacity of older adults be preserved and enhanced? Psychological Science in the Public Interest, 9, 1-65. doi 10.1111/j.15396053.2009.01034.x

Infurna, F., \& Gerstorf, D. (2013). Linking perceived control, physical activity, and biological health to memory change. Psychology and Aging, 28, 1147-1163. doi 10.1037/a0033327

Kozik, P., Hoppmann, C., \& Gerstorf, D. (2014). Future time perspective: Opportunities and limitations are differentially associated with subjective well-being and hair cortisol concentration. Gerontology, 61, 166-174. doi 10.1159/000368716

Lang, F., \& Carstensen, L. (2002). Time counts: Future time perspective, goals, and social relationships. Psychology and Aging, 17, 125-39.

Lindenberger, U. (2014). Human cognitive aging: Corriger la fortune? Science, 346(6209), 572-578. doi 10.1126/science. 1254403

Lindenberger, U., Wenger, E., \& Lövdén, M. (2017). Toward a stronger science of human plasticity. Nature Reviews Neuroscience, 18, 261-262. doi 10.1038/nrn.2017.44

Lisman, J., \& Grace, A. (2005). The hippocampal-VTA loop: Controlling the entry of information into long-term memory. Neuron, 46, 703-713. doi 10.1016/j.neuron.2005.05.002

Lisman, J., Grace, A., \& Duzel, E. (2011). A neo-Hebbian framework for episodic memory: Role of dopamine-dependent late LTP. Trends in Neurosciences, 34, 536-547. doi 10.1016/j.tins.2011.07.006

Maguire, E. A. (2001). The retrosplenial contribution to human navigation: a review of lesion and neuroimaging findings. Scandinavian Journal of Psychology, 42, 225-238.

Maguire, E.A., Spiers, H.J., Good, C. D., Hartley, T., Frackowiak, R. S., \& Burgess, N. (2003). Navigation expertise and the human hippocampus: A structural brain imaging analysis. Hippocampus, 13, 250-259. doi 10.1002/hipo.10087

Mathalon, D. H., Sullivan, E.V., Rawles, J.M., \& Pfefferbaum, A. (1993). Correction for head size in brain-imaging measurements. Psychiatry Research, 50, 121-139.

Mather, M., \& Carstensen, L. (2003). Aging and attentional biases for emotional faces. Psychological Science, 14, 409-415. doi 10.1111/1467-9280.01455

Mather, M., \& Carstensen, L. L. (2005). Aging and motivated cognition: The positivity effect in attention and memory. Trends in Cognitive Sciences, 9, 496-502. doi 10.1016/j.tics.2005.08.005

Mather, M., \& Schoeke, A. (2011). Positive outcomes enhance incidental learning for both younger and older adults. Frontiers in Neuroscience, 5, 129. doi 10.3389/fnins.2011.00129

Muthén, L. K., \& Muthén, B. O. (2010). Mplus user's guide. (6th ed.). Los Angeles: Author.

Nestor, P., Fryer, T., Smielewski, P., \& Hodges, J. (2003). Limbic hypometabolism in Alzheimer's disease and mild cognitive impairment. Annals of Neurology, 54, 343-351. doi 10.1002/ana.10669

Nyberg, L., Kim, A. S., Habib, R., Levine, B., \& Tulving, E. (2010). Consciousness of subjective time in the brain. Proceedings of the National Academy of Sciences of the United States of America, 107, 22356-22359. doi 10.1073/pnas.1016823108

Raz, N., \& Lindenberger, U. (2013). Life-span plasticity of the brain and cognition: From questions to evidence and back. Neuroscience and Biobehavioral Reviews, 37, 2195-2200. doi 10.1016/j.neubiorev. 2013.10.003

Raz, N., \& Rodrigue, K. (2006). Differential aging of the brain: Patterns, cognitive correlates and modifiers. Neuroscience and Biobehavioral Reviews, 30, 730-748. doi 10.1016/j.neubiorev.2006.07.001

Rowe, J.W., \& Kahn, R. L. (2015). Successful aging 2.0: Conceptual expansions for the 21 st century. The Journals of Gerontology, Series B: Psychological Sciences and Social Sciences, 70, 593-596. doi 10.1093/geronb/gbv025

Schacter, D. L., Addis, D. R., \& Buckner, R. L. (2008). Episodic simulation of future events: Concepts, data, and applications. Annals of the New York Academy of Sciences, 1124, 39-60. doi 10.1196/ annals.1440.001

Schacter, D. L., Addis, D. R., Hassabis, D., Martin, V. C., Spreng, R. N., \& Szpunar, K. K. (2012). The future of memory: Remembering, imagining, and the brain. Neuron, 76, 677-694. doi 10.1016/ j.neuron.2012.11.001

Szpunar, K. K., Watson, J.M., \& McDermott, K. B. (2007). Neural substrates of envisioning the future. Proceedings of the National Academy of Sciences of the United States of America, 104, 642-647. doi 10.1073/pnas.0610082104

Van Praag, H., Kempermann, G., \& Gage, F. H. (2000). Neural consequences of environmental enrichment. Nature Reviews $\mathrm{Neu-}$ roscience, 1, 191-198. doi 10.1038/35044558

Vann, S., Aggleton, J., \& Maguire, E. (2009). What does the retrosplenial cortex do? Nature Reviews Neuroscience, 10, 792-802. doi 10.1038/nrn2733

Wheeler, M. A., Stuss, D. T., \& Tulving, E. (1997). Toward a theory of episodic memory: The frontal lobes and autonoetic consciousness. Psychological Bulletin, 121, 331-354.

Whitwell, J. L., Crum, W. R., Watt, H. C., \& Fox, N. C. (2001). Normalization of cerebral volumes by use of intracranial volume: Implications for longitudinal quantitative MR imaging. American Journal of Neuroradiology, 22, 1483-1489.

Wittmann, B., Bunzeck, N., Dolan, R., \& Düzel, E. (2007). Anticipation of novelty recruits reward system and hippocampus while promoting recollection. Neurolmage, 38, 194-202. doi 10.1016/ j.neuroimage.2007.06.038

Manuscript submitted: 01.10.2017

Manuscript accepted after revision: 17.04.2018

Published online: 25.06.2018

\section{Sandra Düzel}

Max Planck Institute for Human Development

Lentzeallee 94

14195 Berlin

Germany

duezel@mpib-berlin.mpg.de 


\section{Appendix 1}

In follow-up analyses, we set up VBM analyses including the SHH-Q Work and SHH-Q Goals factor with the same covariates as described in the main models separately, in order to investigate the dissociation of the four SHH-Q factors regarding their specific or nonspecific association to brain regions. Both VBM analyses revealed no significant associations between the two SHH-Q factor scores and specific brain regions after nonstationary correction (all $p \mathrm{~s}>.005)$.

\section{Appendix 2}

\section{SHH-Q items (translation of the German final version)}

Each SHH-Q item was presented above a timescale ranging from 10 to 110 years.

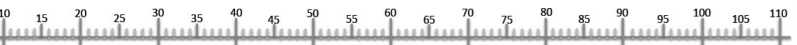

\section{Control Items}

1. Please estimate how old you will approximately become: . . ?

2. Up to which age will you maintain your independence?

3. Please assess up to which age you will feel healthy.

4. From which age do you think you will be chronically sick?

\section{SHH-Q Items on Bodily Fitness (SHH-Q Body)}

5. Up to which age do you think you will be able to maintain your current physical performance?

6. Up to which age do you believe you will be able to maintain or even increase your fitness by training?

7. Up to which age will you be able to incorporate additional physical activity into your daily life (e.g., bicycle tours, Nordic walking, jogging, swimming, working out, hiking)?

8. From which age onward do you think your motivation to do sports will decline?

9. Please judge at what age your physical capabilities will have decreased by more than half (e.g., from which age onward will you only be able to jog, swim, or walk half of the current distance)?

10. Up to which age will you be able to motivate yourself to train more intensively than at present in order to maintain or improve your fitness?

11. Up to which age will you be able to practice sports such as jogging, cycling, Nordic walking, or tennis? Please assess yourself even if you will not actually carry it out.

12. Up to which age will you be able to practice sports such as yoga, pilates, relaxation, gymnastics? Please assess yourself even if you will not actually carry it out.
13. Up to which age will you be able to practice sports such as strengthening muscles or weight training? Please assess yourself.

\section{SHH-Q Items on Occupational Perspectives (Work)}

14. Considering your present health, up to which maximum age could you imagine working?

15. Considering your present health, up to which maximum age could you imagine starting a completely new job?

16. From which age on would cardiovascular complaints hinder you from working in a new occupational field?

17. From which age on would your mental fitness hinder you from working in a new occupational field?

18. From which age on would loss of motivation or a lack of energy hinder you from working in a new occupational field?

\section{SHH-Q Items on Overall Goals and Plans (Life Goals)}

19. How far does the overall planning of your goals reach into the future?

20. How far ahead do you generally plan activities (e.g., visiting your family next month, going on a round-theworld trip in 5 years, learning a new language in the next 10 years)?

21. In view of your physical fitness, up to which age do you generally plan your future ahead?

\section{SHH-Q Items on Novelty-Oriented Exploration (Novelty)}

22. Up to which age do you feel physically and mentally able to learn new sports skills independently (e.g., dancing, sailing, mountaineering, diving)?

23. Up to which age do you feel physically and mentally able to be creative in a new way (e.g., theater group, painting, digital photography, etc.)?

24. Up to which age do you feel physically and mentally able to emigrate to an foreign country or to move to a new place independently?

25. Up to which age do you feel physically and mentally able to learn something mentally new (e.g., learn a new language, extend your computer knowledge, learn new historical facts, participate in a book club, etc.)?

26. Up to which age do you feel physically and mentally able to explore new countries and cultures actively and independently (e.g., city tours, expeditions, round trips)?

27. Up to which age do you feel physically and mentally able to learn new skills independently (e.g., a new craft, juggling)?

28. Up to which age do you feel physically and mentally able to independently come to grips with new technology?

29. Up to which age do you feel physically and mentally able to independently try new things in your daily life (e.g., taking a new route, visiting a new mall)?

30. Up to what age do you think you will be physically and mentally able to meet new people (e.g., by joining a sports group, club, or other interest groups)? 